\title{
IPEG Symposium 1988 Japan
}

September 29th - October 1st, 1988

Kobe International Conference Hall Port-Island, Kobe, Japan

A training course is planned from September 26th to 28th, 1

The main topics for the symposium are:

(1) EEG/EPand

Prognosis of psychiatric diseases

Prognosis of treatment effects

Eye movements and other artifacts

Nootropics

Psychotropic drugs

Performance during recording

Clinical rating of psychiatric diseases

Drug effects in healthy subjects and in patients

Dependence of effects on pretreatment values

Control group definition

Vigilance

Behavior measures and other variables

Blood levels 
(2) EEG/EP topics related to drugs/psychiatry

Event related (late) potentials (cognitive processes)

Mapping methods for data and results

Spatial analysis

Segmentation procedures

Single response recognition/analysis

Automatic sleep staging

(3) Theories of association and dissociation of EEG and behavior in animals and man

(4) Aging and dementia: pharmaco-EEG in drug development

It is planned to allocate $10 \mathrm{~min}$ for free communications and $20 \mathrm{~min}$ for the lectures on the main topics.

The local organization will be handled by:

Masami Saito, MD, DMS Professor and Chairman Department of Neuropsychiatry Kansai Medical University Fumizonocho Moriguchi Osaka 570 (Japan) 
For further information, write to:

Lateiner International Japan KK Mrs. Machiko Tsujino Crescent Plaza 1st Floor 2-4-6 Minami-Aoyama Minato-Ku Tokyo 107 (Japan) 